\title{
FES cycling may promote recovery of leg function after incomplete spinal cord injury
}

\author{
N Donaldson*,1, TA Perkins ${ }^{1}$, R Fitzwater ${ }^{2}$, DE Wood ${ }^{3}$ and F Middleton ${ }^{2}$ \\ ${ }^{1}$ Department of Medical Physics and Bioengineering, University College London, UK; ${ }^{2}$ Spinal Injuries Unit, Royal \\ National Orthopaedic Hospital Trust, Stanmore, UK; ${ }^{3}$ Department of Medical Physics and Bioengineering, Salisbury \\ District Hospital, Salisbury, UK
}

\begin{abstract}
Study design: Single subject pilot.
Objectives: (i) To see whether strength and endurance for recreational cycling by functional electrical stimulation (FES) are possible following spinal cord injury (SCI). (ii) To develop the equipment for FES-cycling.

Setting: England.

Methods: Near-isometric or cycling exercise was performed by the incomplete SCI subject at home.

Results: After training for an average of 21 min per day for 16 months, the stimulated muscles increased in size and the subject was able to cycle for $12 \mathrm{~km}$ on the level. Surprisingly, there was a substantial increase in the measured voluntary strength of the knee extensors and the subject reports improved leg function.

Conclusion: FES-cycling may promote recovery after incomplete spinal cord injury. If so, it offers the possibility of being a convenient method for widespread use.

Sponsorship: Spinal Injuries Unit, Royal National Orthopaedic Hospital, Stanmore. Spinal Cord (2000) 38, 680-682
\end{abstract}

Keywords: spinal cord injury; FES; cycling; recovery; voluntary function; exercise

\section{Introduction}

The effects of spinal cord injury (SCI) due to incomplete lesions may be treated. Three therapies may improve walking speed: drugs and gait training, gait training with partial body weight support, and electrical stimulation. See Barbeau et $a l^{1}$ for a recent review. Dietz et $a l^{2}$ showed that, by initial training with reduced body weight, patients became able to take steps, though the voluntary muscle strengths were not increased. When functional electrical stimulation is used to improve gait, recovery of natural function may be a side effect or 'carry over'. ${ }^{3}$ In 111 hemiplegic stroke patients, who had used tibialis anterior stimulation for 4.5 months to correct dropped foot, Taylor et $\mathrm{al}^{4}$ found a mean increase of $14 \%$ in walking speed of gait without stimulation.

While patients, whose gait is only slightly affected, could use FES for relatively long periods, and might thereby obtain therapeutic benefit, many SCI patients can not. For them, gait training on a treadmill, perhaps with some weight support by a parachute harness, and with therapists to guide the feet, may be successful in improving the gait and eventually allow

*Correspondence: N Donaldson, UCL Department of Medical Physics and Bioengineering, 11-20 Capper Street, London WC1E 6JA, UK independent walking. But this requires the patient to attend a clinic frequently and then the attention of more than one therapist. These requirements will impede widespread use.

The results reported in this Communication arose out of a study with a different purpose. Given the health benefits of FES cycling exercise, ${ }^{5}$ we are interested in whether recreational or sport FES cycling is feasible. We have carried out a pilot study to see whether a volunteer could, with sufficient training, obtain enough strength and endurance for outdoor cycling; also to develop the equipment.

\section{Methods}

At the start of the project, the subject was age 52 with an incomplete lesion at T11/12 from an accident 10 years earlier. Comparing his Frankel scores before his discharge from the spinal injuries unit (1988) to those immediately before FES exercising began (1998), the right side was stable $(49 / 50)$ but the left had deteriorated slightly from $34 / 50$ to $29 / 50$. He could stand and walk short distances with two crutches and a left ankle splint due to the extensor tone $(\mathrm{L}>\mathrm{R})$.

$\mathrm{He}$ was provided with an 8-channel stimulator which could deliver pulses up to $500 \mu$ s and $150 \mathrm{~mA}$ 
that he used either for near-isometric ('armchair') exercising, or cycle training. The electrodes were 3inch diameter round on quadriceps or $3 \times 5$ inch elliptical on hamstring muscles and 2-inch diameter on the shank muscles. The near-isometric exercise was intended to increase endurance; the stimulation was applied to eight muscle groups (see Figure 1, top graph), all of which are now used for cycling but were not used for cycling during the period described here. The $20 \mathrm{~Hz}$ stimulation was applied alternately to the flexors of one leg and extensors of the other leg and then the extensors of the first leg and the flexors of the other. Each phase lasted $12 \mathrm{~s}$. The intensities were set at palpated maximal contraction. Cycling was done on a recumbent tricycle with a left ankle orthosis, fixed to the pedal, to which the foot was strapped. For indoor exercising, the back wheel rested on friction rollers. The stimulator was operated by the method of Carmen Bruck, ${ }^{6}$ in which one switch applies stimulation to left quadriceps and right hamstrings while another switch applies stimulation to right quadriceps and left hamstrings. The subject learnt to use these switches to obtain pedalling action. Stimulation was always at $20 \mathrm{~Hz}$ and the intensities were fixed at palpated maximal contraction levels.

The subject kept a log of FES-isometric exercise, FES-cycling exercise and those occasional sessions when he tried cycling with voluntary muscle contractions only. This record is shown in the figure. He intended to do regular FES exercise but, as can be seen, over this 445-day period, his actual exercising was rather erratic due to the other calls on his time.

\section{Results}

Soon after starting training, he was able to cycle $1.2 \mathrm{~km}$. By 5 months, he was able to cycle on level tarmac at $7.7 \mathrm{~km} / \mathrm{h}$ for $10 \mathrm{~km}$ (about $25 \mathrm{~W}$ of mechanical output), and after 15 months, the distance was $12 \mathrm{~km}$. Measured by ultrasound, the thicknesses of the muscles had increased as follows: right quadriceps, $28 \%$, left quadriceps, $14 \%$, right calf $4 \%$, left calf $14 \%$. Note that because the left calf was only stimulated during isometric training, it received less than half that of the thigh muscles. Knee extension moments ( $\mathrm{Nm}$ with standard errors) were measured before and after training:

$$
\begin{array}{ll}
\text { Stimulation } & \text { Right } 69 \pm 5 \text { to } 102 \pm 7 \\
& \text { Left } 72 \pm 5 \text { to } 96 \pm 7 \\
\text { Voluntary } & \text { Right } 59 \pm 4 \text { to } 86 \pm 6 \\
& \text { Left } 0 \text { to } 34 \pm 3 .
\end{array}
$$

In daily activities, without stimulation, this recovery of voluntary function has allowed him to walk short distances with one crutch, to pick things up from the floor more easily, and for his left leg to move itself when it becomes uncomfortable (he has residual sensation).

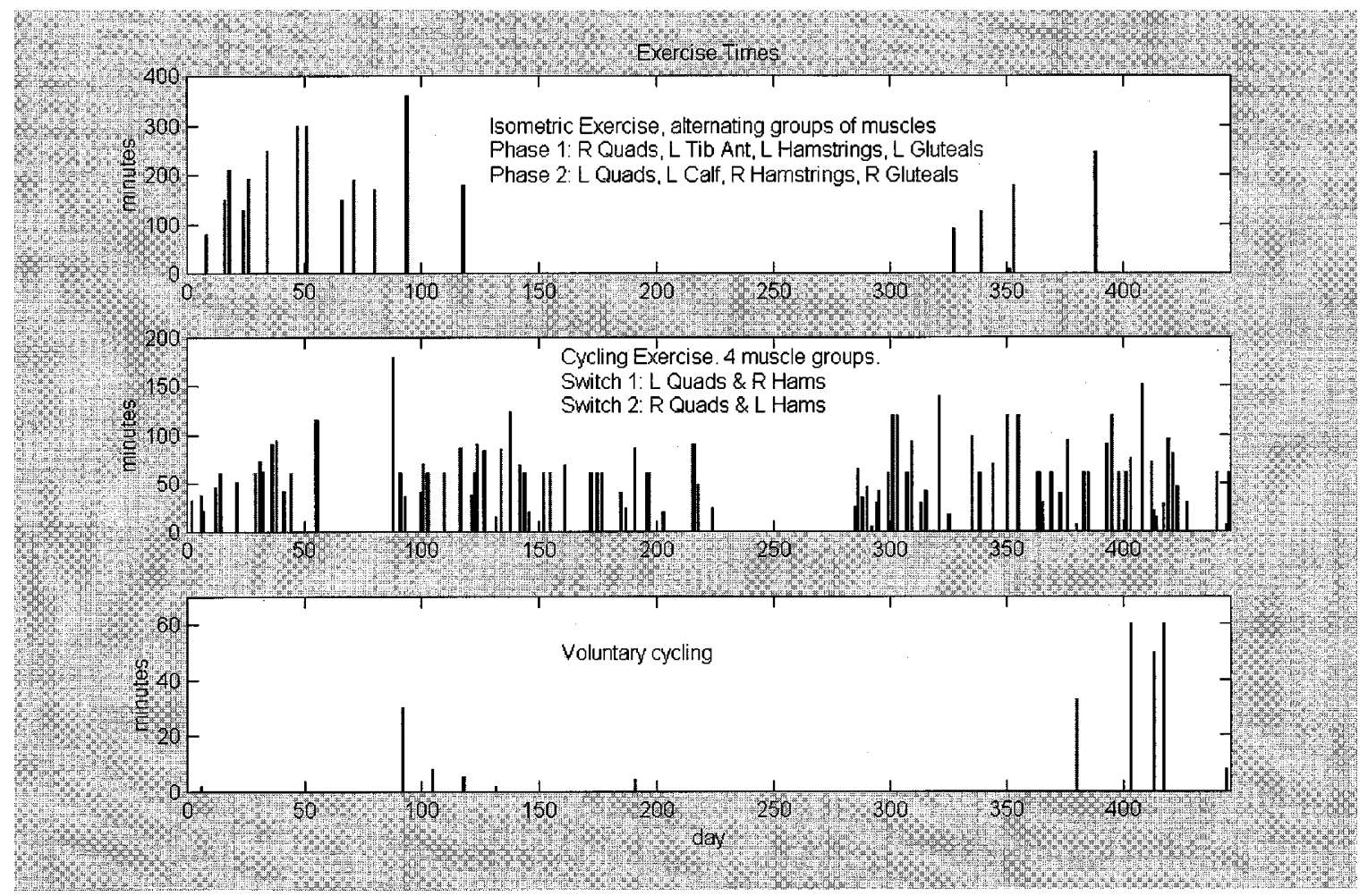

Figure 1 Near-isometric-FES training, FES-cycle training, and voluntary cycling. Log for 16-month period 


\section{Discussion}

After 15 months of FES training, we have found a remarkable recovery of voluntary strength and natural leg function. This can not be explained by the increased muscle strength, at least on the left, because, while the improvement in strength due to stimulus was $33 \%(96 / 72)$, there was negligible initial voluntary strength. It seems unlikely that spontaneous peripheral nerve recovery occurred more than 10 years after injury therefore the cycling exercise caused neuromuscular re-education. This result is, as far as we know, the first report of improved voluntary leg function which is due to FES cycling exercise alone. Sloan et $a l^{7}$ previously reported improved voluntary strength after FES cycling, but $4 / 9$ of their subjects were less than 1 year post-injury so it is not clear whether the recovery they reported was due to cycling exercise or peripheral nerve regeneration. If this recovery is possible for some incomplete SCI patients, it is significant because there is no physical reason why they can not, at no great expense, do this exercise themselves at home under the occasional supervision of a visiting therapist. Such practical issues will be of increasing importance if new methods for cellular repair change complete lesions into incomplete lesions. Then, practical methods of neuro-muscular reeducation may be needed to obtain the desired functional outcome and to measure the improvement.

\section{References}

1 Barbeau $\mathrm{H}$ et al. Does neurorehabilitation play a role in the recovery of walking in neurological populations? Ann NY Acad Sci 1998; 860: $377-392$.

2 Dietz V, Columbo G, Jensen L, Baumgartner L. Locomotor capacity of spinal cord in paraplegic patients. Ann Neurol 1995; 3: $574-582$.

3 Bajd T, Kralj A, Stefancic M, Lavrac N. Use of functional electrical stimulation in the lower extremities of incomplete spinal cord injury. Artif Org 1999; 23: $403-409$.

4 Taylor PN et al. Clinical use of the Odstock dropped foot stimulator. Its effect on the effort and speed of walking. Arch Phys Med Rehabil 1999; 80: $1577-1583$

5 Janssen TWJ, Glaser RM, Shuster DB. Clinical efficacy of electrical stimulation exercise training: effects on health, fitness, and function. Topics Spinal Cord Injury Rehabil 1998; 3: 33-49.

6 Tomorrow's World, BBC1 TV, November 4th, 1998.

7 Sloan KE et al. Musculoskeletal effects of an electrical stimulation induced cycling programme in the spinal injured. Paraplegia 1994; 32: 407-415. 